\title{
El sistema de innovación design driven como modelo estratégico y la propuesta de política nacional de diseño para Chile 2007
}

\section{Resumen}

Durante el primer semestre de 2007 la autora en su calidad de Coordinadora Ejecutiva de la Comisión participó en la creación de una Propuesta de Política Nacional de Diseño para Chile. La propuesta fue presentada al Ministro de Economía de la época Sr. Alejandro Ferreiro, el 27 de julio del mismo año, en el evento MIPYME DISEÑA bajo el lema "Innovar, Crecer, Competir".

Esta propuesta de política pública se articuló teniendo como base conceptual y metodológica el Sistema de Innovación Design_driven (SID_d), modelo estratégico e instrumental que permite mapear y articular los habilitantes de una política de Innovación Design_driven. El SID_d está constituido por los actores, las actuaciones, los proyectos, la dimensión espacio-temporal, el modelamiento de procesos de Innovación Design_driven genera un sistema clasificatorio que permite caracterizar -al menos- tres tipologías: I) SID_d nivel avanzado, 2) SID_d nivel intermedio y 3 ) SID_d nivel emergente. Por otra parte, este modelo como instrumento estratégico permite visualizar un modelo ideal para países en vías de desarrollo y es útil como referente para la propuesta de estrategias que fortalezcan el desarrollo, proyección y consolidación del Diseño en SID_d del tipo emergente. Este modelo fue desarrollado por la autora como resultado de su trabajo de tesis doctoral entre los años 2004 y 2006 y considera los resultados de la investigación realizada sobre cuatro casos europeos, España, Portugal, Italia y Finlandia, en los cuales el Diseño es parte del Sistema Nacional de Innovación.

Este artículo explica la configuración del Sistema de Innovación Design_Driven (SID_d) como modelo estratégico base para el desarrollo del Diseño en países emergentes y revisa los principales componentes de la citada propuesta de Política Nacional de Diseño. 
1.- El "Sistema de Innovación Design_driven" (SID_d)

En los últimos 25 años, cambios estratégicos en la agregación de valor han sido generados mediante la articulación de un sistema que integra la economía productiva, la gobernanza y la gestión del conocimiento. Dependiendo del país o región es posible encontrar diversas denominaciones, pero en general se ha consensuado en llamarle Sistema Nacional de Innovación (SIN) ${ }^{(1)}$.

En los países más desarrollados el Diseño tiene una participación activa en los procesos de innovación ${ }^{(2)}$ a través de la acción proyectual creativa. Esta participación del Diseño en el Sistema Nacional de Innovación mediante procesos de 'Innovación Design_driven' (3) (ID_d) es sustentado por el "Sistema de Innovación Design_driven" (SID_d).

Este SID_d se articula a partir de tres componentes:

I) El sistema económico productivo, materializa la oferta de productos y servicios en una perspectiva teleológica de maximización del margen y la rentabilidad.

2) La sistema $\mathrm{I}+\mathrm{D}+\mathrm{i}$, que desde la gobernanza gestiona el conocimiento generando instancias de coordinación público-privadas, movilización de distintas capacidades del aparato público y sus recursos, en función de programas estratégicos gubernamentales. En forma paralela, pone en marcha dinámicas de gestión del conocimiento $(\mathrm{I}+\mathrm{D}+\mathrm{i})$ en el contexto de los procesos de innovación a nivel país, y

3) El sistema proyectual-creativo denominado "Sistema Diseño"(4), que se define como un conjunto de actores relevantes y sus interrelaciones que soportan al Diseño como disciplina profesional para operar al interior de una comunidad, país o región $n^{(s)}$.En el contexto de las tendencias culturales globales y locales territoriales este sistema -por su génesis cultural- tiene la capacidad de generar nuevos significados y códigos formales para productos y servicios.

Las interrelaciones entre estos tres sistemas y las prioridades ponderadas que cada uno de ellos presenta respecto de los otros han sido materia del diseño estratégico con el cual los distintos países han abordado sus desafíos, en el contexto de su naturaleza cultural, política, social y económica, entre otras variables.

Por otra parte, el SID_d representa una opción de carácter pragmático, al momento de implementar estrategias de fomento productivo en los distintos países, puesto que indexa la matriz de productos y servicios de un país con las tendencias culturales globales, en el contexto del patrimonio natural y cultural de los distintos territorios y clusters locales.
En el campo de la investigación realizada en países que presentan distintos niveles de profundidad en políticas de innovación, se ha detectado que los más avanzados han otorgado una preponderancia mayor al Sistema Diseño. Esto debido a que en sus estrategias han priorizado el fenómeno cultural, como un fenómeno que determina las tendencias en las prácticas de consumo al interior de los mercados globales y locales. Una referencia para esta opción de estrategia país es Finlandia, donde la innovación blanda o de significado ha sido el puntapié inicial del posterior desarrollo innovativo-tecnológico, teniendo hoy día el mismo nivel de importancia que la innovación dura o tecnológica(s).

También se ha observado que en aquellos casos donde el Sistema Diseño tiene un mayor nivel en cuanto a alcances, contenidos y dinámicas creativas, el sistema productivo y la $\mathrm{I}+\mathrm{D}+\mathrm{i}$ genera saltos en las capacidades competitivas en el contexto de los mercados globales y locales, en el mediano y largo plazo.

El 'Sistema de Innovación Design_driven', entonces, se define como un sistema interconectado con el Sistema Nacional de Innovación, Sistema Económico-productivo y Sistema Diseño. Este sistema soporta el desempeño del Diseño en los procesos de innovación orientado a la creación y agregación de identidad-valor de productos y servicios que llegan al mercado y/o comunidad. El SID_d considera los requerimientos económicos, tecnológicos, culturales y sociales definidos tanto por las políticas públicas como por el mercado ${ }^{(6)}$.

\subsection{Los dominios institucionales del SID_d}

Desde un punto de vista estrictamente teórico, el SID_d es un sistema heterogéneo constituido por elementos que provienen de tres dominios o universos presentes en la realidad de un país o región.

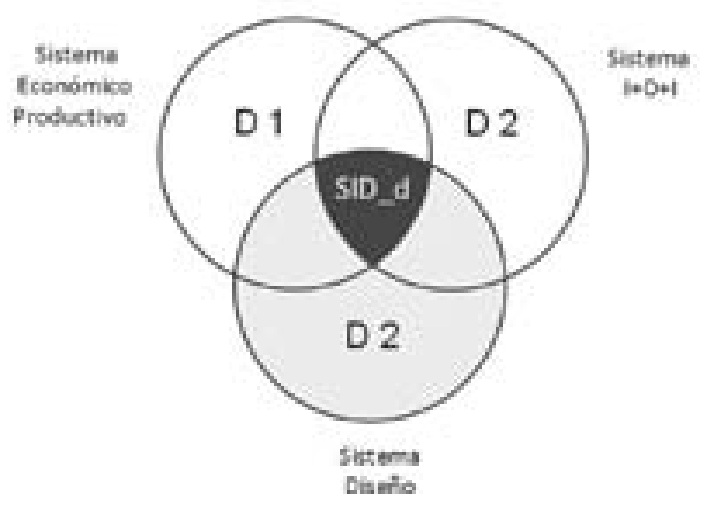

Figura I.

Los tres dominios institucionales del SID_d. Fuente: Mollenhauer, K., 2007 
Estos dominios o universos que componen el SID_d son: a) El Sistema Económico-productivo (Di), b) El Sistema de la I+D+i o Sistema de Innovación Nacional $\left(\mathrm{D}_{2}\right)^{(8)}$ y c) El 'Sistema Diseño' $\left(\mathrm{D}_{3}\right)$. En cada uno de estos sistemas se identifican elementos comunes a los tres sistemas y vinculados entre sí, los cuales encuentran un punto de intersección común a los tres dominios, conformando de este modo el 'Sistema de Innovación Design_driven' (SID_d).

\subsection{Los componentes institucionales del SID_d}

En el contexto de la materialización de las estrategias para el desarrollo económico, los países estudiados han creado mecanismos que ponen en marcha conocimientos, recursos y energía con el fin de sustentar las políticas de innovación.

Se ha podido observar, entonces, que es común a todos los SID_d

Figura 2.

La estructura del SID d estudiados el hecho de que los proyectos son el resultado de las diFuente: Mollenhauer, $K$.,

2007

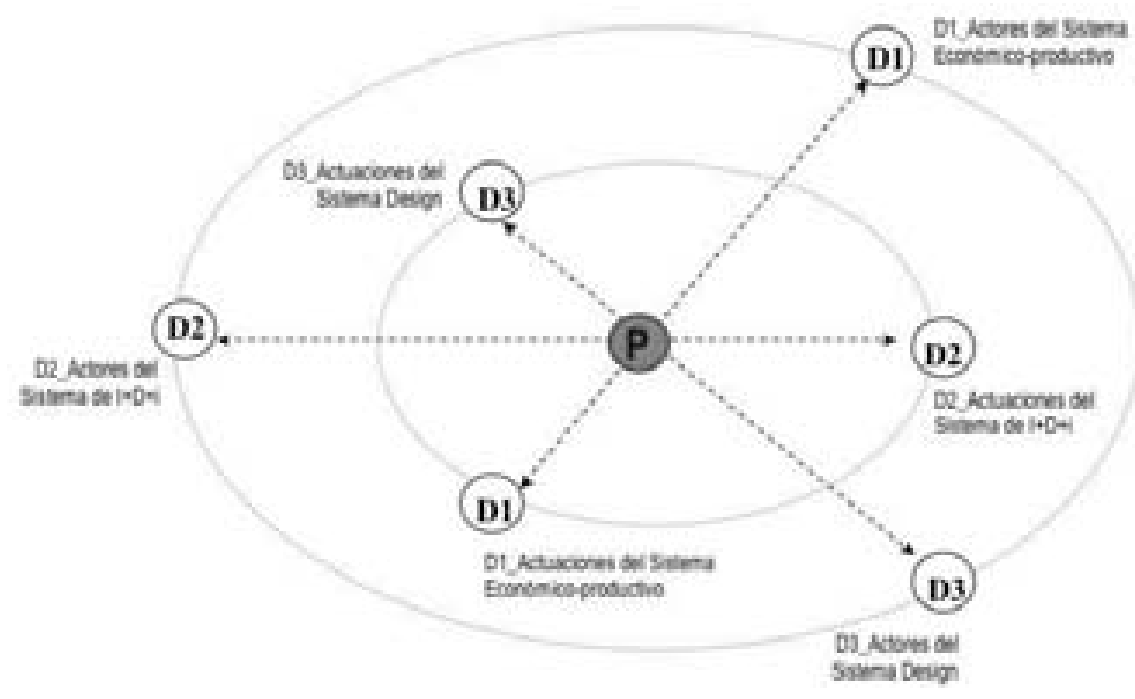

Cuando estos componentes han estado presentes en los países estudiados, éstos han obtenido un resultado de mayor alcance e impacto, toda vez que han integrado de forma coherente y sistémica a: I) actores públicos y privados, 2) actuaciones políticas, estratégicas e instrumentales, 3) proyectos reconocidos como referentes, 4) un horizonte proyectos y 5) el modelamiento de buenas prácticas.
1.2.1. Sistema de Actores Institucionales de Innovación Design_driven El Sistema de Actores Institucionales de Innovación Design_driven (ID_d) estructura, demanda, gestiona, ejecuta, divulga y promueve las actuaciones y proyectos de soporte a la 'Innovación Design-driven'. Estos actores institucionales son entes pertenecientes a los siguientes sectores: a) Industrial-empresarial; b) Gobierno y administración; c) Investigación, desarrollo e innovación (I+D+i); d) Formación; e) Profesión y f) Promoción del Diseño. Este último actor, de la promoción del Diseño, es el que da los lineamientos, articula y desarrolla el sistema en su conjunto.

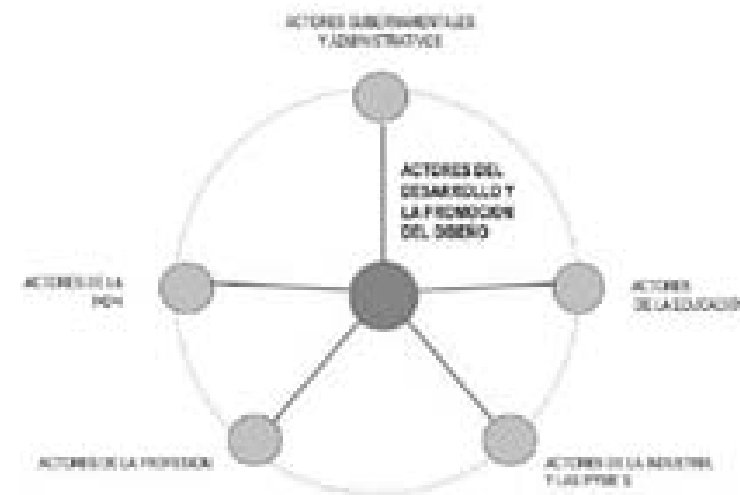

Figura 3 Modelo del Sistema de Actores.

Fuente: Mollenhauer, $K$, 2007

1.2.2. Sistema de Actuaciones Institucionales de Innovación Design_driven

El Sistema de Actuaciones Institucionales de ID_d dinamiza las acciones llevadas a cabo por el conjunto de actores que dan origen a los proyectos, otorgando un marco institucional que soporte a la Innovación Design-driven (ID_d) como actividad dentro del Sistema de Innovación Nacional con un fuerte impacto en el Sistema Económico Productivo.

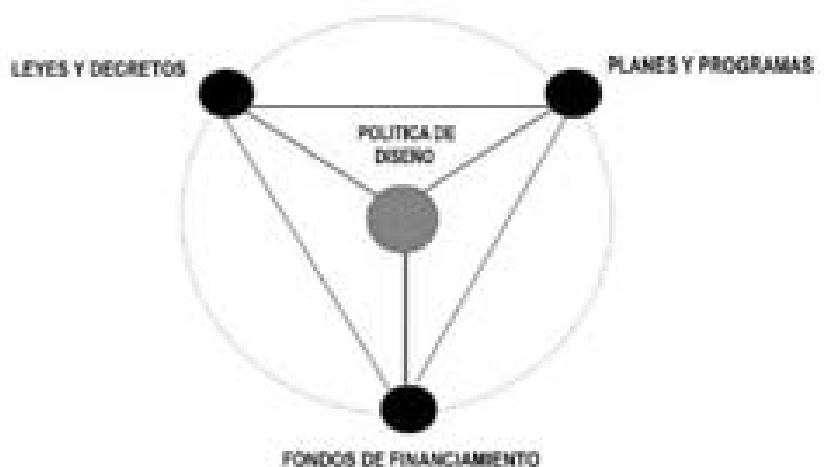

Figura 4 Modelo del Sistema de Actuaciones. Fuente: Mollenhauer, $K$., 2007 
Estas actuaciones institucionales son: a) Leyes o decretos (nacionales y/o regionales); b) Planes o Programas institucionales; c) Instrumentos de financiamiento; y d) Política (nacional y/o regional). Esta última, la política de Diseño, es la actuación que define los objetivos y establece los lineamientos estratégicos que estructuran y organizan el resto de las actuaciones y articulan a los actores para el desarrollo de proyectos de Innovación Design_driven.

Sistema de Proyectos Institucionales de Innovación Design_driven

El Sistema de Proyectos Institucionales de ID_d es el resultado de las dinámicas de interrelación entre el sistema de actores y de actuaciones, que conscientemente llevan adelante acciones proyectuales con criterios propios de la Innovación Design_driven, considerados como referentes de buenas prácticas y susceptibles de ser sistematizados como modelos instrumentales o metodologías.

En este sistema es posible encontrar, al menos, cuatro tipos de proyectos que son la base del modelamiento de metodologías de Innovación Design_driven:

I) del tipo I+D, vinculada con la investigación básica (Tipo A); 2) del tipo Transferencia de 'Know-how', vinculada con la investigación aplicada (Tipo B); 3 ) del tipo de Plan Piloto, vinculado a la experimentación (Tipo C); y 4) del tipo de Promoción, vinculado a la difusión (Tipo D). Estos proyectos a su vez se agrupan en dos cate-

Figura 5. gorías mayores de proyectos: a) de implantación del Diseño (AyB), Modelo del Sistema de y b) de sensibilización hacia el Diseño (CyD).

Proyectos del SID_d. Los tipos de proyectos son albergados en Programas e InstrumenFuente: Mollenhauer, $K ., \quad$ tos que hacen operativa la Política.

2007

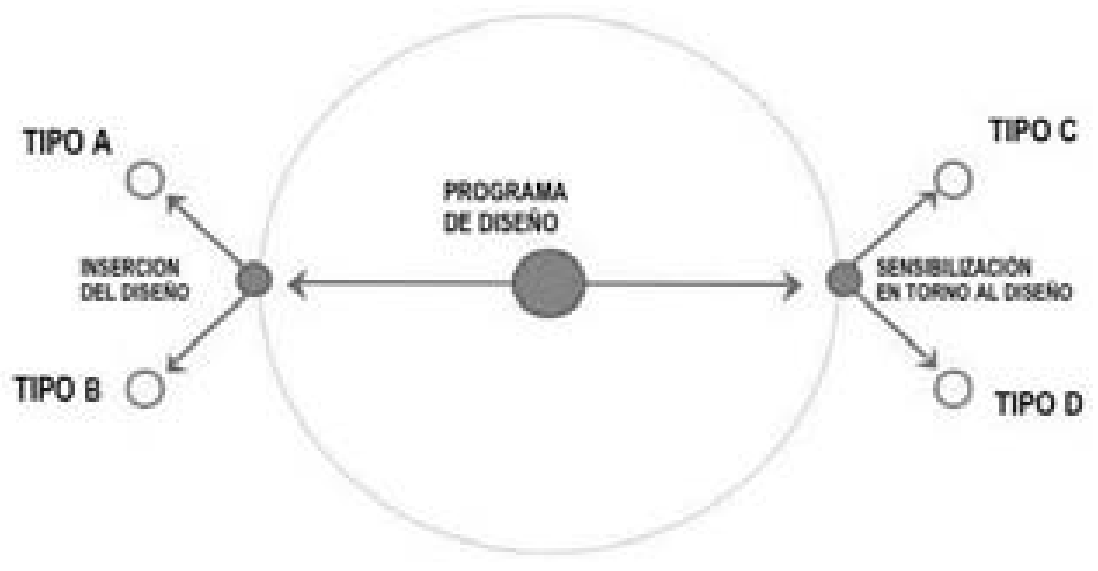

1.2.3. Sistema Espacio-tiempo de la Innovación Design_driven El Sistema Espacio-temporal de la ID_d engloba y sitúa temporalmente a las actuaciones como los componentes que estructuran, dinamizan y producen los resultados de la ID_d, en tres periodos: a) las actuaciones pasadas; b) las actuaciones presentes; y c) las actuaciones futuras.

Asociados a estos tres momentos, es posible identificar tres hitos temporales del SID_d: I) momento pasado o Génesis; 2) momento presente o Estado del Arte; y 3 ) momento futuro o Tendencia.

A partir de estos tres hitos en la línea espacio-tiempo del SID_d, Figura 6. es posible entonces identificar cuatro periodos para la Política: I) Modelo espacio-tiempo. Periodo de Gestación de la Política, 2) Periodo de Desarrollo de la Fuente: Mollenhauer, $K$., Política; 3 ) Periodo de Consolidación de la Política; y 4) Periodo de 2007. Proyección de la Política.

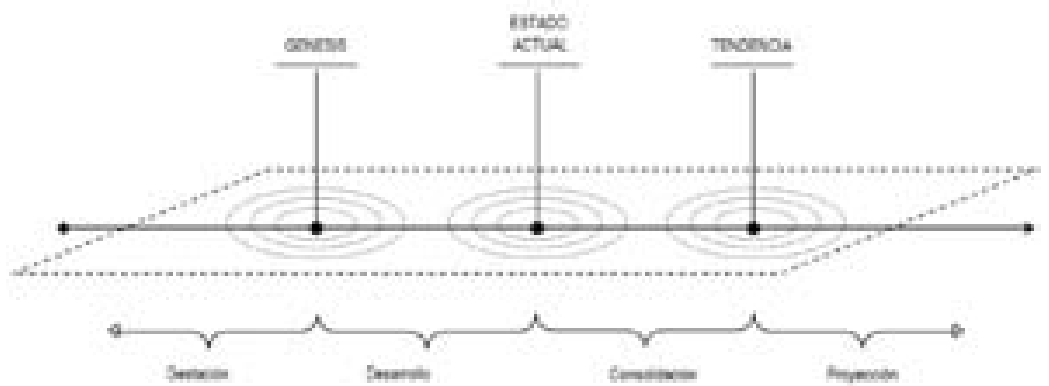

La definición de estos cuatro periodos y tres hitos permite realizar análisis diacrónicos y sincrónicos de los actores, de las actuaciones y de los proyectos pudiendo estudiar el pasado, para comprender el presente y poder planificar el futuro. De este modo, el SID_d configura su dimensión estratégica y metodológica para la gestación, desarrollo, consolidación y proyección de una Política de Diseño a partir de un proceso sistemático de mejora continua.

\subsubsection{Sistema de Modelamiento de Metodologías \\ de Innovación Design_driven}

El Sistema de Modelamiento de Metodologías de ID_d tiene su origen en la sistematización de las buenas prácticas de procesos de ID_d y la construcción de modelos instrumentales que permitan orientar estratégicamente a los actores, priorizar las actuaciones y definir el conjunto de proyectos que aterrizan la Política de Diseño.

Estos modelos instrumentales o metodologías de ID_d son diversos y dependen del grado de madurez del SID_d. 
2. El SID_d "ideal" como modelo de referencia para la consolidación y proyección del Diseño en países en vías de desarrollo El SID_d como modelo instrumental permite, entre otras cosas, visualizar la génesis de una Política de Diseño para países en vías de desarrollo, ya que perfila los contenidos fundamentales para orientar un desarrollo tecnológico culturalmente sostenible ( ). Esta visualización es posible a partir del "SID_d ideal".

El "SID_d ideal" es el modelamiento sistémico de las mejores prácticas observadas en el estudio de caso realizado y se constituye en un modelo de referencia que orienta la Política Nacional de Diseño para Chile ( ) a partir de los siguientes componentes tipológicos: I) Tipos de Sistemas, II) Tipos de Roles, y III) Tipos de Impactos.

I) Tipología de Sistemas: los tipos de sistemas son cuatro y organizados dentro del SID_d como sistemas interconectados. Incluyen: a) Seis tipos de actores o stakeholders provenientes del sector del Gobierno \& Administración, de la Educación, de la I+D \& de la Transferencia de Tecnología, de la Profesión, de la Industria \& Empresa, de la promoción, todos en su conjunto constituyen el Sistema de Actores; b) Cuatro tipos de actuaciones como son las políticas, leyes o decretos, planes y programas, instrumentos de fomento, todos constituyen el Sistema de Actuaciones; c) Ocho tipos de proyectos guiados por el diseño y clasificados en proyectos de investigación y desarrollo, proyectos de investigación aplicada y transferencia, proyectos de sensibilización, proyectos de redes colaborativas, proyectos educativos, proyectos de consultorías de Diseño, proyectos de patrimonio histórico, proyectos de mejora continua, y que en su conjunto constituyen el Sistema de Proyectos; y d) Diversos tipos de metodologías de intervención para proyectos de ID_d. Estos cuatro sistemas y sus componentes dan origen a una tipología de roles.

II) Tipología de Roles: los tipos de roles determinan la utilidad instrumental y estratégica del SID_d en tanto logran un desarrollo tecnológico culturalmente sostenible. Un Sistema Diseño determinado puede contribuir a este tipo de desarrollo si es capaz de articular en un todo los cinco roles que lo definen: a) Rol Económico-productivo, b) Rol Tecnológico, c) Rol Sociocultural, a) Rol Ético y a) Rol Político. La conjugación y ejecución de estos roles en un todo sistémico generaría un conjunto de impactos en torno a la misión de una Política de Diseño en los países en vías de desarrollo, los cuales pueden influir a la hora de querer impactar positivamente la sociedad.
III) Tipología de Impactos: a lo anterior es posible agregar una tercera tipología, referida al tipo de impacto de los actores, actuaciones, proyectos, metodologías y roles ya mencionados. Estos se relacionan con: a) el desarrollo del Diseño, b) la consolidación del Diseño, y c) la proyección del Diseño.

\begin{tabular}{|c|c|c|c|}
\hline & Tipología & Roles del Diseño & Impacto \\
\hline Proyectos & $\begin{array}{l}\text {-Educación } \\
\text {-Sensibilización } \\
\text {-Investigación básica } \\
\text {-Investigación aplicada } \\
\text {-Redes colaborativas } \\
\text {-Consultorías de Diseño } \\
\text {-Mejora continua } \\
\text {-Patrimonio histórico }\end{array}$ & $\begin{array}{l}\text { Rol Económico- } \\
\text { productivo } \\
\text { Rol Tecnológico } \\
\text { Rol Sociocultural }\end{array}$ & $\begin{array}{l}\text { En el desarrollo del } \\
\text { Diseño (basado } \\
\text { en la generación } \\
\text { de Know-how, } \\
\text { de Innovación, } \\
\text { de Cultura y de } \\
\text { Patrimonio de } \\
\text { Diseño) }\end{array}$ \\
\hline Actuaciones & $\begin{array}{l}\text {-Instrumentos de } \\
\text { financiamiento } \\
\text {-Planes y Programas } \\
\text {-Políticas } \\
\text {-Leyes y decretos }\end{array}$ & Rol Político & $\begin{array}{l}\text { En la consolidación } \\
\text { del Diseño } \\
\text { (basado en la } \\
\text { institucionalización } \\
\text { del mismo) }\end{array}$ \\
\hline Actores & $\begin{array}{l}\text {-Educación } \\
\text {-Profesión } \\
\text {-Promoción } \\
\text {-I+D \& Transferencia } \\
\text { Tecnológica } \\
\text {-Estado \& administración } \\
\text {-Industria \& empresa }\end{array}$ & Rol Ético & $\begin{array}{l}\text { En la proyección del } \\
\text { Diseño (basada en } \\
\text { la misión de éste) }\end{array}$ \\
\hline
\end{tabular}

ElSID_d como modelo ideal se presenta como una constelación de Tabla r. Matrizde actores, actuaciones, proyectos, metodologías y roles en un todo un SID_d 'ideal'como articulado y sistémico, cuyo impacto esperado es el 'desarrollo tec- modelo instrumental y nológico culturalmente sostenible’ de los terceros países. estratégico para un 'desa-

En síntesis, el "SID_d ideal” es un modelo instrumental o meto- rrollo tecnológico cultudología, por cuanto puede contribuir al desarrollo del Diseño en una ralmente sostenible. comunidad, país o región. Esta posibilidad de desarrollo se basa en Fuente:Mollenhauer, K., los distintos tipos de resultados institucionales que constituyen el 2007. Sistema de Proyectos, los que favorecen al posicionamiento del Diseño a través de su rol económico-productivo, tecnológico y sociocultural, contribuyendo así al desarrollo de: a) una Cultura de Diseño, b) un Patrimonio de Diseño, c) un Know-how de Diseño y d) una Innovación guiada por el Diseño, cumpliendo con su misión instrumental para un desarrollo tecnológico culturalmente sostenible.

También el SID_d como modelo 'ideal' potencia su dimensión estratégica, toda vez que puede contribuir a la consolidación del Diseño. Esta posibilidad radica en los distintos tipos de acciones institucionales que constituyen el Sistema de Actuaciones, las cuales 


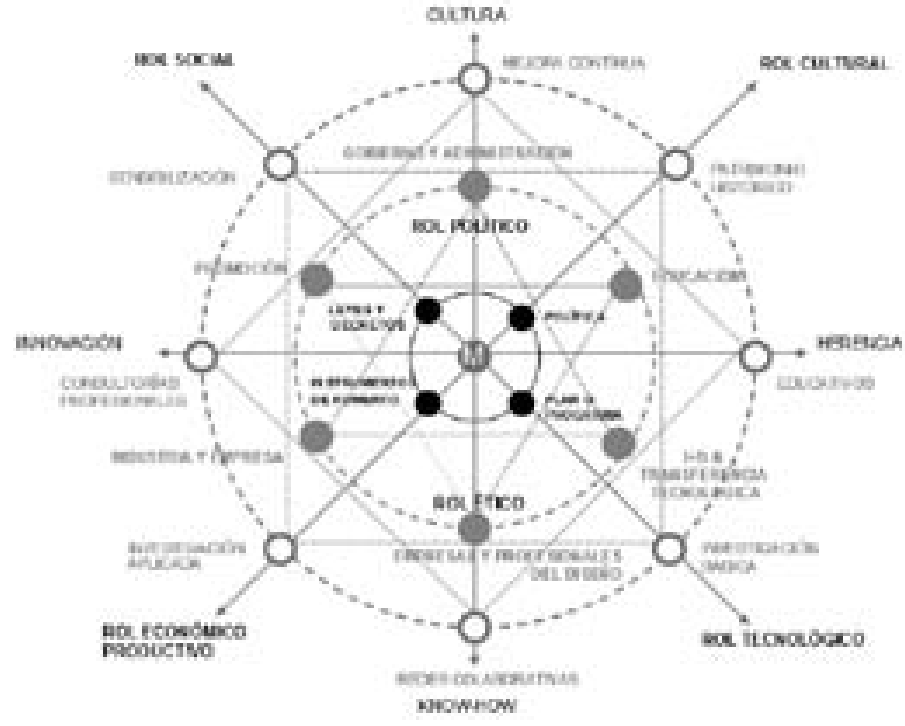

Figura 7.

Modelo de un "SID_d

ideal".

Fuente: Mollenhauer, $K$.

2007 . contribuyen a que el Diseño cumpla con su misión institucional, a través del rol Político, permitiendo así la consolidación de su misión a través de la institucionalización de su Sistema de Actores.

El SID_d como modelo 'ideal' contribuye a la proyección del Diseño, toda vez que desde el ejercicio de su rol ético puede velar por el impacto culturalmente sostenible que tiene el Sistema de Proyectos, como expresión pragmática de su misión económicoproductiva, tecnológica, social y cultural.

\section{Sistemas de Innovación Design_driven del tipo Emergen- tes, Intermedios y Avanzados}

El SID_d es un modelo instrumental clasificatorio que permite 'mapear' el estado de situación del Sistema Diseño de cualquier país o región en cuanto a la profundidad de sus estrategias de desarrollo, el grado de madurez con el cual aborda los roles que tiene y su vinculación con las políticas de innovación y las dinámicas concretas que se ponen en movimiento en relación a esta opción estratégica.

Para este mapeo se realiza un análisis del estado del arte y de la proyección de los componentes de: I) los Sistemas Institucionales (actores, actuaciones, proyectos, dimensión espacio-temporal y modelamiento de metodologías); 2) los Roles que el Diseño tiene en esa comunidad; y 3) los Impactos reales y potenciales del Diseño en el desarrollo de los sectores económico-productivo, tecnológico, social y cultural de cada país o región y se comparan con los componentes del SID_d "ideal”. Las conclusiones de este análisis comparativo permiten establecer un sistema clasificatorio a través de cual es posi- ble caracterizar - al menos- tres tipologías respecto de la profundidad que presentan los SID_d en cada país. Estas categorías son: I) SID_d avanzado, 2) SID_d intermedio y 3) SID_d emergente.

El SID_d como modelo instrumental estratégico permite no sólo establecer los componentes fundamentales necesarios para la gestación, desarrollo, consolidación y proyección del Diseño en países en vías de desarrollo basado en la visualización de un "modelo ideal", sino que también permite 'mapear' el estado de situación del Diseño -en relación a su injerencia dentro de los Sistemas de Innovación y Sistemas Económico-productivos- de cualquier país o región

\subsection{SID_d del tipo avanzado}

Este tipo de SID_d es el más completo e incorpora un gran número de actores, actuaciones y proyectos evidenciando la madurez que ha alcanzado el Sistema Diseño en el contexto de las Políticas públicas asociadas al desarrollo económico-productivo, tecnológico, social y cultural.

En el Sistema de Actores Institucionales encontramos ocho tipos. Aquellos provenientes del sector del gobierno y administración, de la educación, de la I+D, de la Transferencia de Know-how, de la Profesión, de la Industria \& Empresa, de la promoción, más el sector de la Memoria. En los países desarrollados donde el Diseño ha logrado consolidarse institucionalmente el Sistema Diseño se ocupa del patrimonio histórico del Diseño o, dicho en otras palabras, de la memoria del Diseño como parte de la estrategia para proyectar la disciplina hacia el futuro, basándose en la puesta en valor de los roles del Diseño y de la misión que éstos han cumplido en los distintos momentos de la historia.

En el Sistema de Actuaciones Institucionales radica la fortaleza del tipo de SID_d avanzado. Aquí encontramos un Sistema de Actores Institucionales completo, el cual ha logrado institucionalizar el Diseño como agente protagonista en el desarrollo nacional. También encontramos una Política Nacional de Diseño, la cual es producto de una decisión política, de gobierno y de Estado y por tanto su creación emana de un decreto o ley institucional. Por lo mismo, esta Política cuenta con planes, programas e instrumentos propios que propician la sistematización de las buenas prácticas de procesos de ID_d y la construcción de metodologías estructuradas que permiten orientar estratégicamente a los actores, priorizar las actuaciones y definir el conjunto de proyectos que aterrizan la Política de Diseño. 


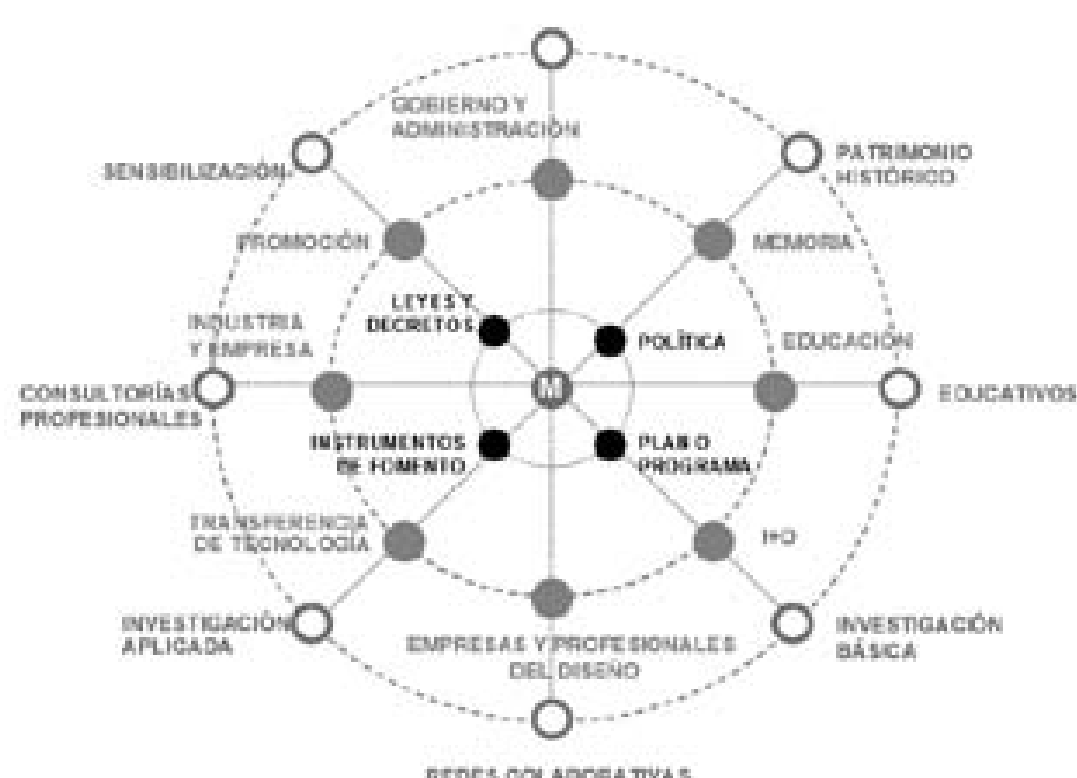

proce golaOCGA mVAS

Figura 8 Modelo de un Como consecuencia de lo anterior, el Sistema de Proyectos InstituSID_d avanzado. Fuente: Mollenhauer, $K$., 2007 cionales se ve fortalecido por el hecho de disponer de un Sistema de Actuaciones con programas e instrumentos propios para el financiamiento de la Política de Diseño, habilitando así al Sistema de Actores y potenciando el Sistema de Proyectos. Así nos encontramos con los ocho tipos de proyectos clasificados en: proyectos de investigación y desarrollo, proyectos de investigación aplicada y transferencia, proyectos de sensibilización, redes colaborativas, proyectos educativos, consultorías de Diseño, proyectos de patrimonio histórico, mejora continua. Esta diversidad tipológica potencia al Sistema Diseño permitiéndole su desarrollo, consolidación y proyección.

\subsection{SID_d del tipo intermedio}

Este tipo de SID_d está definido por una presencia menor de actores, actuaciones y proyectos que en el caso del SID_d avanzado.

Esto tiene su origen en el hecho de que el Sistema de Actuaciones Institucionales solo dispone de dos de los cuatro tipos que es posible encontrar en un SID_d avanzado. En este Sistema de Actuaciones Institucionales no existen Políticas de Diseño, ni leyes, ni decretos que institucionalicen el Diseño como articulador de políticas públicas. Existen algunos planes y programas, que a través de instrumentos de financiamiento permiten tangencialmente la participación del Sistema Diseño en los procesos de Innovación. En este tipo de SID_d el Diseño es mencionado escasamente en políticas más generales como el Plan de Innovación Nacional o los Programas de apoyo a las PYME's, etc., donde los instrumentos que incorporan acciones de Diseño son escasos y le restan protagonismo, por tanto, el Sistema de Actores y de Proyectos se ve debilitado.

Es así como en el Sistema de Actores Institucionales encontramos siete tipos provenientes del sector del gobierno y administración, de la educación, de la I+D, de la Transferencia de Know-how, de la Profesión, de la Industria y la empresa, de la promoción, faltando aquel actor encargado del rescate, construcción y registro de la memoria del Diseño, lo que en definitiva afecta la proyección del Sistema Diseño.

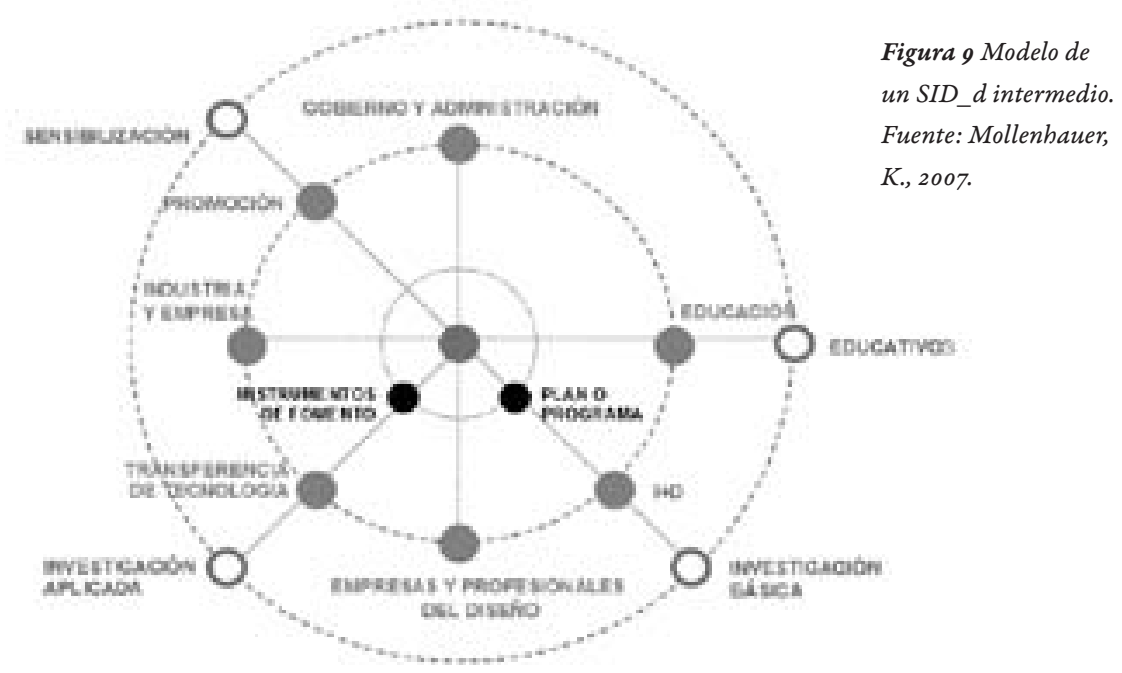

Por otra parte, el Sistema de Proyectos Institucionales cuenta básicamente con cuatro tipos de proyectos: I) educación, 2) sensibilización, 3) investigación básica y 4) investigación aplicada. Si bien esto puede parecer contradictorio en cuanto a que poseen el sistema de actores prácticamente completos y, por lo tanto, deberíamos pensar que el tipo de proyectos debiera ser amplio, la capacidad de gestión de los mismos queda reducida por la falta de metodologías estructuradas de ID_d que permitan orientar estratégicamente a los actores y priorizar las actuaciones traduciéndose en la falta de instrumentos destinados para este tipo de iniciativas. Por lo tanto, el ejercicio del Diseño no queda claramente identificado. Esto influye en que los fondos disponibles a los cuales el Diseño puede optar provienen de iniciativas generales vinculadas a las políticas de ciencia y tecnología o a los 
programas de innovación, no siendo éstos, no aplica instrumentos "con nombre y apellido de Diseño", con lo cual la participación del Sistema Diseño se ve debilitada.

\subsection{SID_d del tipo emergente}

El SID_d del tipo emergente está definido por una escasa presencia de actores, actuaciones y proyectos debilitando mucho el Sistema de Roles y el Sistema de Impactos que el Sistema Diseño puede tener en los países o regiones de este tipo.

En el Sistema de Actores encontramos sólo tres tipos: I) del sector de la Educación pues el Diseño tiene sus orígenes en este sector, 2) le sigue el sector de la profesión como consecuencia lógica del sector de la Educación, y 3 ) el sector de la Industria y la Empresa con un bajo nivel de participación como expresión de una incipiente demanda por profesionales de Diseño. Referidos al Sistema de Actuaciones, encontramos sólo un tipo, el cual se refiere básicamente a instrumentos de financiamiento que no están vinculados a planes y programas relacionados directamente con el Diseño, es decir, que no se desprenden de un Programa o Política de Diseño Industrial y mucho menos de un decreto o ley que la institucionalice. Tampoco encontramos metodologías estructuradas de ID_d, sólo podemos reconocer una práctica no sistematizada, la que en general redunda en una escasa valoración del proceso de diseño, afectando significativamente los salarios del sector Diseño.

Finalmente referido al Sistema de Proyectos, los tipos emergentes cuentan básicamente con los proyectos provenientes del sector de la Educación y que si bien pueden ser intentos de relacionarse con el sector de la Industria-Empresa y/o con el Estado, son generalmente un ejercicio académico el cual queda relegado a la experimentación.

Con el SID_d como sistema clasificatorio es posible mapear la realidad en la cual operan los Sistemas Design de países y regiones, y establecer las estrategias necesarias para avanzar en el desarrollo, consolidación y proyección de Sistemas Design ${ }^{(\mathrm{II})}$.

En síntesis, el SID_d surge como un subsistema interrelacionado dentro del Sistema de Innovación Nacional, Sistema Económicoproductivo y Sistemas Design considera el conjunto de elementos que, en el ámbito nacional, regional o local, pueden actuar e interactuar, a favor o en contra, de cualquier proceso de creación, difusión, o uso de conocimiento económicamente útil. De la interrelación entre los actores y las actuaciones surgen los proyectos del SID_d, los cuales pueden contribuir a establecer fortalezas desde los altos niveles de los actores nacionales y regionales de la política, la economía, la cultura y la educación, al identificar y validar aquellas dinámicas guiadas por

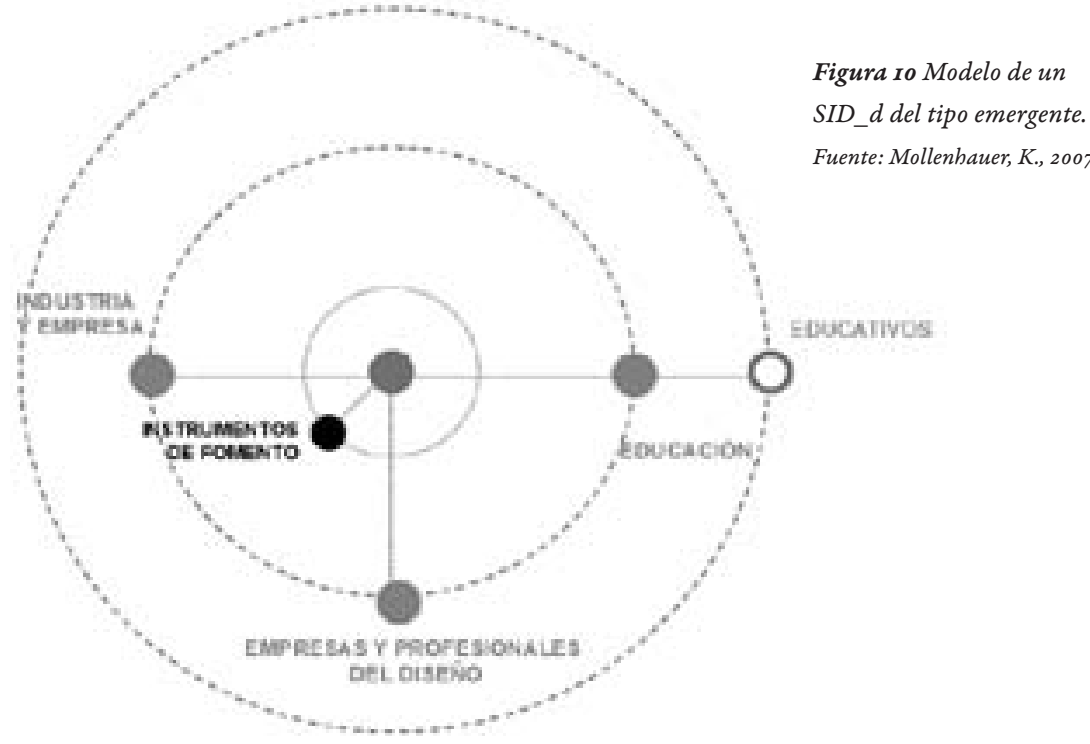

el Diseño. Por lo tanto, desde una visión estratégica, el Diseño queda en posición de influir en los procesos de innovación de un país o región, toda vez que los actores del 'Sistema Diseño', orientados fundamentalmente por la Innovación Design_driven, participan en los procesos impulsados por el Sistema de Innovación y el Sistema Económicoproductivo. De este modo, el SID_d se constituye en un modelo instrumental estratégico a partir del cual es gestar, desarrollar, consolidar y proyectar Políticas de Diseño en países y regiones. En el caso de Chile, en el año 2007, el SID_d orientó la estructuración de la propuesta de Política Nacional de Diseño.

\section{Propuesta de una Política Nacional de Diseño para Chile 2007}

Las políticas de Diseño han significado un componente clave en las estrategias que los países desarrollados han adoptado para alcanzar mayores niveles de actividad económica. Despegues significativos en el ámbito comercial han sido obtenidos en base a una conducción coherente de la política pública mediante un sistema que privilegia la agregación de valor sobre la oferta productiva de estos países. Un ejemplo de este singular fenómeno ha sido Finlandia, que ha logado cambios estratégicos significativos en la agregación de valor mediante un sistema que integra la 'proyectualidad' creativa, la economía productiva y la gobernanza y gestión del conocimiento.

Durante el primer semestre del año 2007, un conjunto de actores del mundo del Diseño en Chile se cita para reflexionar acerca de los 
fundamentos conceptuales y teóricos sobre los cuales trabajar por una Política Nacional de Diseño ${ }^{(12)}$, conformando así la Comisión Nacional para la Propuesta de una Política Nacional de Diseño para Chile ${ }^{(13)}$.

Como parte del proceso de Investigación se trabajó en base a documentos realizados por los distintos miembros de la comisión y a ejemplos de casos de éxito en materias de políticas y de Innovación Design_driven. Las conclusiones arrojadas por el estudio de base sobre el Sistema Diseño Chileno establecieron la necesidad de gestar y desarrollar una Política de Diseño nacional, así como de articular coherentemente a los stakeholders (o actores principales) del Sistema. Para ello, y desde un punto de vista metodológico, fue el 'Sistema de Innovación Design_driven' (SID_d) el modelo instrumental que dio estructura interna y visión estratégica al proceso de creación de esta propuesta de Política Nacional de Diseño 2007. Este modelo instrumental estableció criterios a través de los cuales el equipo pudo trabajar de forma conjunta y coordinada estableciendo como punto de partida los siguientes habilitantes:

\subsection{Componentes de la Política Nacional de Diseño} para Chile 2007

Los propósitos de esta Propuesta de Política son siete y guardan relación con:

I) Fomentar la participación del gobierno;

2) Integrar el Diseño dentro del sector industrial y empresarial;

3) Innovar a través de la investigación aplicada en Diseño;

4) Formar con modelos innovadores que promuevan el Diseño como estrategia;

5) Promover una cultura de Diseño;

6) Desarrollar el Diseño como sector económico con impacto del PIB nacional; y

7) Mejorar y fortalecer continuamente el Sistema Diseño Chile.

Los sectores estratégicos que intenta abordar la Propuesta están relacionados con los propósitos mencionados anteriormente y son: Gobierno, Empresa, Investigación, Educación, Promoción, Profesión, Coordinación General. A su vez, estos sectores estratégicos y sus propósitos están vinculados con los actores institucionales estratégicos. Estos actores son:

I) Subcomité del Diseño (vinculado al sector Gobierno y a Fomentar);

2) Subdirección programas de Diseño (vinculado al sector Empresa y a Integrar);

3) Consejo Nacional de Investigación en Diseño (vinculado al

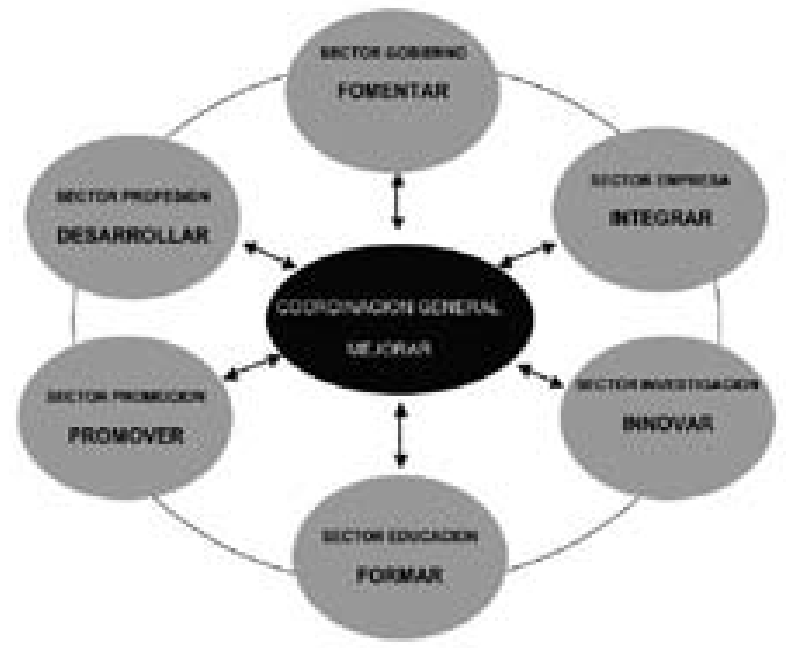

Figura 1 II.

Propósitos y sectores estratégicos.

Fuente: Mollenhauer, $K$, Rodríguez, A., et al; 2007

sector Investigación y a Innovar);

4) Consejo Nacional de Escuelas de Diseño (vinculado al sector Educación y a Formar);

5) Centro Nacional de Promoción del Diseño (vinculado al sector de la Promoción y a Promover);

6) Consejo Nacional de entidades gremiales y empresariales del Diseño (vinculado al sector de la Profesión y al Desarrollo de la misma);

7) Consejo Nacional de Diseño (vinculado a la Coordinación General y a la Mejora Continua).

\subsection{Modelo de Gestión de la Política}

El modelo de Gestión de la Política establece para los siete propósitos, los siete sectores estratégicos y los siete actores mencionados anteriormente, un total de siete principios.

Estos principios son:

I) El apoyo del Gobierno central y los gobiernos regionales es fundamental para la integración del Diseño en el Sistema Nacional de Innovación, aportando de este modo a las estrategias nacionales y regionales de competitividad.

2) La construcción de una confianza empresarial desde y hacia el Diseño, a través de la mejora de la percepción que los empresarios tienen del Diseño, resulta fundamental.

3) El Diseño debe y puede contribuir a los procesos de innovación en Chile.

4) El aumento de las competencias de los Diseñadores median- 
te la educación continua y de nivel internacional, así como una estrecha cooperación entre los sectores educacionales y empresariales, contribuyen en la producción de elevados estándares de conocimiento en torno a los requerimientos de Diseño.

5) Construir conocimiento sobre el Diseño para alcanzar el reconocimiento de su dimensión estratégica como factor clave de diferenciación.

6) El desarrollo del Diseño como sector económico con impacto en el PIB nacional.

7) Otorgar una perspectiva estratégica al desarrollo, consolidación y proyección de la Política Nacional de Diseño.

Instituciones relacio-

nadas con los sectores $y$

actores estratégicos del

modelo.

Fuente: Mollenhauer, $K$.

Rodríguez, A., et al; 2007.

Cada uno de estos principios y todos en conjunto dan origen a 28 objetivos, 24 programas y sus correspondientes instrumentos para la implementación ${ }^{(14)}$, los cuales deben ser articulados por al menos una decena de organismos público-privados existentes o nuevos relacionados con las distintas materias mencionadas.

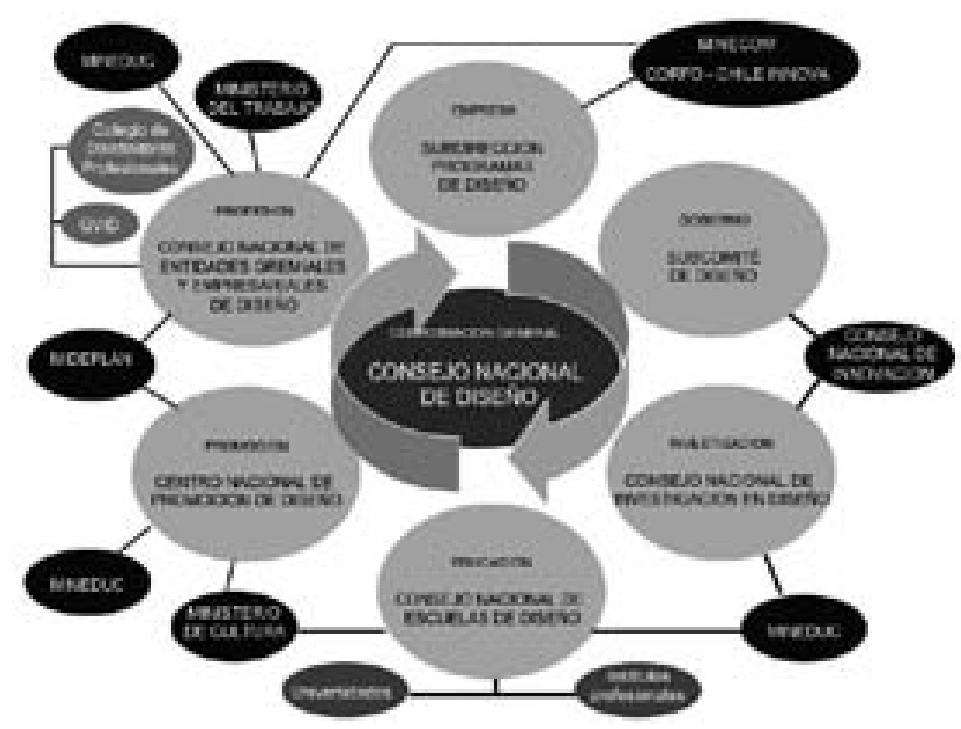

\subsection{Estrategia de Implementación}

La estrategia de implementación considera una perspectiva espaciotemporal organizada en cuatro periodos o etapas: I) Etapa de gestación; 2) Etapa de desarrollo; 3 ) Etapa de consolidación; y 4) Etapa de proyección.

\section{Conclusiones}

Para comprender a cabalidad la lógica interna que rigió la creación de la Propuesta de Política Nacional de Diseño 2007 ha sido necesario conocer el modelo del "Sistema de Innovación Design_driven" (SID_d).

Desde esta perspectiva, la posibilidad que ofrece el SID_d, como 'mapa' del estado de situación del Sistema-design vinculado con los Sistemas de Innovación y Sistemas Económico-productivos, permite generar un sistema clasificatorio y comparativo que nos permite hacer un seguimiento de la evolución de los Sistema-design de los países y avanzar hacia un Sistema de Indicadores de Innovación Design_driven. También la lectura de los casos estudiados permite concluir acerca del aprendizaje, por una parte, en torno a la relación entre la existencia de una Política de Diseño y la calidad de los Sistemas de Innovación Nacional, y por otra, respecto de la existencia de una Política de Diseño y del rol institucionalizado del Diseño dentro de los SIN, expresado en la articulación sistémica de los actores, actuaciones, proyectos y metodologías insertos en una dimensión espacio-temporal vinculados con la Innovación Design_driven. De cara a los países en vías de desarrollo y a sus SID d, la posibilidad de pasar de un nivel emergente a uno intermedio depende fundamentalmente del Sistema de Actuaciones Institucionales, ya que es éste el que fortalecerá el Sistema de Proyectos, a través de la institucionalización del Sistema de Actores Institucionales. Es la existencia o inexistencia de una Política Nacional o Regional de Diseño el elemento determinante para que un SID_d sea clasificado como emergente, intermedio o avanzado.

Desde esa misma perspectiva, al revisar los componentes de la Propuesta de Plan Nacional de Diseño para Chile se reconoce al SID_d como base del modelamiento de los habilitantes de la Política. Y si analizamos los propósitos, sectores y actores estratégicos, los principios y el modelo de gestión de la Propuesta de Plan Nacional de Diseño para Chile reconocemos la utilidad del SID_d como modelo estratégico para la elaboración de Políticas Públicas de Diseño.

\section{Epílogo}

Si bien la propuesta de Política Nacional de Diseño 2007 descrita en este artículo no prosperó y, por lo tanto, no se logró la institucionalidad que el Sistema Diseño Chileno requería para avanzar en su grado de madurez, sí sentó un precedente y dejó un mapa de contenidos estratégicos sobre el cual era posible trabajar a futuro.

En el año 20II, el Consejo Nacional de la Cultura y las Artes (CNCA) define al Diseño como parte de las Industrias Culturales y crea el 
Sector Diseño. Este espacio institucional ha sido liderado en su calidad de Coordinador del Sector Diseño por el Sr. Manuel Figueroa, quien en sus dos años de gestión ha llevado adelante un exhaustivo programa de trabajo, gestando y desarrollando muchos de los habilitantes mencionados en la propuesta del 2007, contribuyendo de manera significativa al fortalecimiento del Sistema Diseño chileno.

\section{Notas}

1. Para dar cuenta de las múltiples relaciones y estructuras involucradas en la innovación, suele utilizarse el concepto de Sistema Nacional de Innovación (SNI), al que Manuel Agosin y Neandro Saavedra definen como "el conjunto integrado de actores e instituciones que, en el ámbito de una nación, se interrelacionan con el fin de llevar adelante el proceso de innovación". Véase en Mollenhauer, K. (octubre 2007): "Sistema de Innovación Design_driven. Modelo instrumental para un desarrollo tecnológico culturalmente sostenible", tesis inédita para optar al grado de doctor, Universidad de Barcelona, Cataluña, España, pg. 188.

2. Cabe hacer presente que el Manual de Oslo, en su tercera edición, en los parágrafos 162- 169 -172 -334 -344 y varios otros consigna que el Diseño se debe considerar como parte integrante del desarrollo y de la introducción de innovaciones de productos ( bienes y servicios) y que su intervención puede también constituir innovaciones en mercadotecnia como es la introducción de cambios significativos en la forma, aspecto, etiquetado o envase de productos con el fin de captar un nuevo segmento del mercado . Véase en Manual de Oslo, Guía para la Recogida de Datos e Interpretación de Datos sobre Innovación, Tercera edición; años 2005-2006, publicación conjunta de la OCDE y Eurostat.. Subsecretaría de Economía, Fomento y Reconstrucción, (2005) INNOVAR en Chile. Programa de Innovación Tecnológica, primera edición, Santiago de Chile.

3. Innovación Design_driven (ID_d) es el concepto con el que el Politécnico di Milano define a aquella innovación de 'know-how en la que no sólo cobra valor la innovación funcional y prestacional, sino que también aquella de significado. La 'Innovación Design_driven' significa innovación 'guiada por el Diseño' y es una innovación del tipo proyectual, la cual se relaciona con los procesos de reconfiguración en la creación del valor como resultado del encuentro entre potencialidad técnica y la potencialidad social; un encuentro que para tener lugar necesita de una particular capacidad de adaptarse dentro de diversos universos disciplinarios, organizativos y lingüísticos. Esta relación se da en cuanto existe una relación entre diseño y empresa en el marco de una contínua investigación sobre nuevos perfiles de usuarios y de los productos y servicios necesarios para satisfacerlos. La 'Innovación Design_driven', por lo tanto, hace del Diseño y de su dimensión estratégica una herramienta útil en el desarrollo de nuevos significados para mejorar procesos, formas de comunicación y comercialización de productos, servicios, sistemas y/o experiencias. Véase en ZURLO, F.: CAGLIANO, R.

SIMONELLI, G.; VERGANTI, R. (2002) Innovare con il design: il caso del settore dell'illuminazione in Italia, Editorial 24 ore, Milano.Para dar cuenta de las múltiples relaciones y estructuras involucradas en la innovación, suele utilizarse el concepto de Sistema Nacional de Innovación (SNI), al que Manuel Agosin y Neandro Saavedra definen como "el conjunto integrado de actores e instituciones que, en el ámbito de una nación, se interrelacionan con el fin de llevar adelante el proceso de innovación".

4. El origen de este concepto proviene del Politecnico di Milano donde el Sistema Diseño se denomina Sistema Design. En BERTOLA, P.; SANGIORGI, D.; SIMONELLI, G. (2002): Milano Distretto del Design. Un sistema di luoghi, attori e relazioni al servizio dell'innovazione, II Sole 24 ore, S. p. A, Milano.Sistema de l+D+i, Sistema de Innovación Nacional o Sistema Nacional de Innovación, son todos nombres que se utilizan en distintas partes del mundo para referirse al conjunto de 'stakeholders' que participan dentro de la matriz de la Investigación, el desarrollo y la transferencia de tecnología y 'knowhow necesaria para el desarrollo nacional de un país.

5. MOLLENHAUER, K. (2007): "Sistema de Innovación Design_driven. Modelo instrumental para un desarrollo tecnológico culturalmente sostenible", tesis inédita para optar al grado de doctor, Universidad de Barcelona, Cataluña, España, pg. 190.

6. Op. Cit. pg. 121

7. Ibid. pg. 192

8. Sistema de l+D+i, Sistema de Innovación Nacional o Sistema Nacional de Innovación, son todos nombres que se utilizan en distintas partes del mundo para referirse al conjunto de 'stakeholders' que participan dentro de la matriz de la Investigación, el desarrollo y la transferencia de tecnología y 'knowhow' necesaria para el desarrollo nacional de un país. $\mathrm{N}$ de la $\mathrm{A}$.

9. Se entiende por desarrollo tecnológico culturalmente sostenible al desarrollo de procesos de know-how que contribuyen a las mejoras o desarrollos de nuevos productos o servicios mediante la agregación de valor basada en nuestra cultura. Esto presupone una responsabilidad concreta por parte del Estado y del sector público-privado que debe contribuir a la incorporación de los micro, pequeños y medianos productores a la modernidad, sin que ello implique una pérdida de la identidad local. La experiencia en otros países que se han visto sometidos a situaciones similares indica que esos productores experimentan progresos notables en su productividad e ingresos cuando acceden a los medios. Por otra parte, es necesario trabajar por un desarrollo tecnológico, tal y como lo hacen en la actualidad la mayoría de las naciones, pero sin olvidarnos de que ese desarrollo no puede sobrepasar al centro de la finalidad misma, es decir, al ser humano. Así como tampoco puede sobrepasar al entorno natural y material donde este ser humano habita, pues sino entonces, el hombre como individuo y la humanidad como conjunto deja de ser el centro de dicha finalidad y se constituye en un instrumento del mismo desarrollo con las consiguientes y actuales consecuencias. Una Política de Diseño para el desarrollo tecnológico, donde la tecnociencia avanza a pasos desmesurados y se constituye en el motor de la cultura material, no puede prescindir de unos criterios éticos y políticos que le sustenten, y en esto el Diseño chileno como disciplina tiene mucho que decir, pero por sobre todo mucho por hacer $\mathrm{N}$ de la $\mathrm{A}$.

10. La construcción del SID d como modelo instrumental comienza con los hallazgos provenientes de un benchmarking entre Lombardía y Cataluña realizado durante el 2004 y 2005. Después de esto -en el año 2006- más información sobre las 'buenas prácticas' fue recolectada desde el Caso Portugal. Finalmente, con el fin de 
configurar un modelo 'ideal' fue necesario, por tanto, buscar una realidad que tuviera su SID d totalmente operativo incluyendo el Sistema de Actuaciones Institucionales. Durante el segundo semestre de 2006, Finlandia fue escogida para este cometido por el estado de madurez de su Sistema Design. Véase en

MOLLENHAUER, K.: Op. Cit.

11. Un ejemplo de la utilidad del SID_d como modelo tipológico se puede observar en Ios resultados del mapeo de cinco casos: Chile, Portugal, Lombardía, Cataluña y Finlandia, realizado entre los años 2004 y 2007. En ese entonces se pudo establecer que: a) Chile, era del tipo Emergente (+), por cuanto a la fecha se había iniciado un proceso de gestación de Política Nacional de Diseño y el Sistema Design se encontraba activado. A la fecha la situacion chilena ha cambiado mucho; b) Portugal, era del tipo Intermedio (-), ya que si bien tenía un Sistema de Actores y Proyectos bastante maduro, hasta esa fecha no había señales de una Política 0 Plan Nacional que institucionalizara la labor del Centro Português do Design; c) Lombardia, era del tipo Intermedio (-), ya que si bien el Sistema de Actores era fuerte y el Diseño estaba muy vinculado a la actividad productiva, éste no contaba con fondos exclusivos para la l+D y la Transferencia de Tecnología de Diseño, es decir, el Sistema de Actuaciones no había logrado institucionalizarse a través de un Programa o de Instrumentos de fomento propios; d) Cataluña, era es del tipo Intermedio (+), y resultaba un caso muy particular, por cuanto, si bien el Sistema de Actores estaba completo y existían financiamientos exclusivos para el Diseño, y por tanto debiera ser del tipo Avanzado (-), su sistema educativo universitario (contrariamente a la mayoría de países) no había logrado ser reconocido; es decir a esa fecha el Diseño no era reconocido como una carrera universitaria con grado de licenciado: e) Finlandia era y sigue siendo del tipo Avanzado (+) pues tiene un Sistema de Actores, de Actuaciones y de Proyectos completo y un Sistema Design maduro con más de 130 años de tradición. La Politica Nacional de Finlandia en su primer periodo dispuso de casi 30 millones de euros. Finlandia aún tiene una tarea pendiente relacionada con el desarrollo de un 'Sistema de Evaluación' que le permita medir el impacto y cuantificar el beneficio de la incorporación del Diseño al desarrollo país. Véase en MOLLENHAUER, K: Op. Cit. Y tambíen en Mollenhauer, K. and Arquilla (2006) "V. Paralleli: Lombardy \& Catalonia. Different methods, actor and actions to support SME development by Industrial Design interventions between regions", In International Committee of Design History and Studies ICDHS. 5th Conference and Nordic Forum for Design History Symposia eds. CONNECTING a conference on the multivocality of design history and design studies, University of Art and Design Helsinki, August 29th-29th, Helsinki.

12. La realización de este estudio estuvo a cargo de un equipo de expertos, provenientes del sector de la educación, la profesión y la promoción del Diseño Chileno quienes se constituyeron en la Comisión para la Propuesta de una Política Nacional de Diseño. Esta comisión trabajó durante el periodo de abril a julio de 2007 y estuvo constituida por Patricio González, José Korn, Félix Maldonado, Katherine Mollenhauer, Julio Mejías, Enrique Montero, Alejandro Rodríguez, Diego Rodríguez, Christian Téllez, Ramiro Torres, Rita Soto y Ariel Valdés. La coordinación científica del documento estuvo a cargo de Katherine Mollenhauer y Alejandro Rodríguez. La coordinación ejecutiva de la Comisión recargó en Katherine Mollenhauer. Véase en Mollenhauer, K.; Rodríguez, A., et al (2007): "Propuesta de una Política Nacional de Diseño para Chile", CORFO, Santiago. Obtenido en octubre de 2012 desde http://colegiodisenadores.cl/instrumentos/
13. Los objetivos de la Comisión se centraron en: 1 . Concluir en torno a los antecedentes históricos existentes respecto de la disciplina y la profesión del Diseño en Chile, como un área del conocimiento que ha estado gestándose y desarrollándose a lo largo de los últimos 40 años, y que hoy llega a un estado de madurez suficiente para plantear una Política Nacional de Diseño; 2. Identificar y analizar las 'buenas prácticas' de otros países que han tenido éxito en la implementación de Políticas Nacionales de Diseño y que, por lo tanto, se constituyen en ejemplos emblemáticos a la hora de afrontar un proceso como éste; 3. Identificar y concluir en torno a las actuales oportunidades que existen para el Diseño en Chile, a través de un análisis del sector MIPE y de los instrumentos de financiamiento que hoy día están disponibles para soportar la Innovación Design_driven; y 4. Concluir en torno a una propuesta que sirva de orientación para la elaboración de una Política Nacional de Diseño, así como de los programas e instrumentos necesarios para su soporte e implementación. Véase en Mollenhauer, K.; Rodríguez, A., et Op. Cit.

14. El documento in extenso de la Propuesta de Política Nacional de Diseño con los 28 objetivos, 24 programas y sus correspondientes instrumentos es posible encontrarlo en http://colegiodisenadores.cl/instrumentos/ 


\section{Libros}

Arquilla,V. \& Simonelli, G. \& Vignati, A. (2005). Design, imprese, distretti. Un approccio allinnovazione. Milano: Edizione POLI.design.

Bertola, P. \& Sangiorgi, D., \& Simonelli, G. (2002): Milano Distretto del Design. Un sistema di luoghi, attori e relazioni al servizio dell'innovazione. Milano: Il Sole 24 ore.

Korvenmaa, P. (2006) "Respect", Finnish Design Yearbook 2006. Finland: In Design Forum.

Maffei, S. \& Simonelli, G. (2002) I territori del Design. Made un Italy e sistema productivi locali. Milano: Editorial Il Sole 24 ore.

Zurlo, F. \& Cagliano, R \& Simonelli, G. \& Verganti, R. (2002) Innovare con il design: il caso del settore dellilluminazione in Italia. Milano: Editorial 24 ore.

\section{Artículos}

Korvenmaa, P. (2006). Design, Innovation and National Development: Finland 1995-2005”. In Design Policy as civil action. Post-conference workshop, University of Art and Design Helsinki, August.

Korvenmaa, P. (200I). Rhetoric and Action: Design policies in Finland at the beginning of the third Millennia. In Scandinavian Journal of Design History, II.

Mollenhauer, K. and Arquilla (2006). V. Paralleli: Lombardy \& Catalonia. Different methods, actor and actions to support SME development by Industrial Design interventions between regions", In International Committee of Design History and Studies ICDHS. 5 th Conference and Nordic Forum for Design History Symposia eds. CONNECTING a conference on the multivocality of design history and design studies, August.

Mollenhauer, K. (2007). Design project as a politic act and design policy as a civil action. The case of Chile: Miettinen, Satu (ed). Social Design Actions.

Mollenhauer, K. and Korvenmaa, P. (2007). Designing the Designer: The responsibilities of education in the national/regional system of Design: International Conference on Engineering and Product Design Education, (September) I3-I4.

Mollenhauer, K. and Martí J.M. (2006). Design project as a politic act and design policy as a civil action. Chilean case: In International Committee of Design History and Studies ICDHS. 5 th Conference and Nordic Forum for Design History Symposia eds. Design Policy as civil action. Post-conference workshop, University of Art and Design Helsinki, August.

\section{Documentos}

Collina, L. (2002). El Diseño como sistema. El caso de Milán. Ponencia presentada en Seminario Diseño en la pequeña y mediana empresa: el modelo italiano, Santiago, Chile.

Korvenmaa, P.(1998). Muotoiltu Etu I-II. SITRA (title translated is Designed Advantage), Helsinki, Finlandia

Mollenhauer, K. (2007, octubre). Sistema de Innovación Design_driven. Modelo instrumental para un desarrollo tecnológico culturalmente sostenible. "Tesis inédita para optar al grado de doctor,"Universidad de Barcelona, Cataluña, España.

OCDE. (2005-2006). Manual de Oslo, Guia para la Recogida de Datos e Interpretación de Datos sobre Innovación: Oslo, Noruega.

Simonelli, G. (2002). Diseño y PYMEs. El modelo Italiano. "Seminario (inédito)": Santiago, Chile.

Subsecretaría de Economía, Fomento y Reconstrucción (2005). INNOVAR en Chile. Programa de Innovación Tecnológica: Santiago, Chile.

Transversal Consultores. (2006, mayo). Las regiones de Chile ante la ciencia, tecnología e innovación: diagnósticos regionales y lineamientos para sus estrategias. "Documento asesoría”: Santiago, Chile.

\section{Documento on line}

Mollenhauer, K.; Rodríguez, A., et al. (2007). Propuesta de una Política Nacional de Diseño para Chile, Recuperado en octubre de 20I2, desde http://colegiodisenadores.cl/instrumentos/ 